\title{
Potencial tintóreo de cinco especies de macromycetes silvestres nativos de Tabasco, México, sobre fibras de origen natural
}

\section{Dyeing potential of five species of native wild macromycetes from Tabasco, Mexico, on fibers of natural origin}

\author{
Santa Dolores Carreño Ruíz, Silvia Cappello García, Manuel Antonio García-García, Perla I. Xicoténcatl \\ Maldonado \\ Universidad Juárez Autónoma de Tabasco, División Académica de Ciencias Biológicas, Laboratorio de Micología. Carretera \\ Villahermosa-Cárdenas km 0.5 S/N, Entronque a Bosques de Saloya, C.P. 86150Villahermosa, Tabasco, México.
}

\section{RESUMEN}

Antecedentes: Los tintes naturales se han utilizado desde épocas prehispánicas. Sin embargo, existe poca literatura en la extracción de tintes de hongos para teñir fibras orgánicas.

Objetivo: Evaluar el potencial tintóreo de cinco hongos silvestres sobre fibras de origen vegetal y animal.

Métodos: Los hongos fueron fragmentados, hidratados 24 h, y hervidos por 45 min para obtener los tintes. Se usaron fibras de lino, henequén, estropajo, cáñamo y lana para evaluar la efectividad del tinte. Las fibras se lavaron con jabón neutro, se mordentaron, y se hirvieron por $1 \mathrm{~h}$ en el tinte. En un segundo experimento se modificó el pH de los tintes con vinagre, carbonato de sodio y amoniaco para probar nuevos colores sobre las fibras.

Resultados y conclusiones: Los tintes mostraron tonalidades de café rojizo obscuro a café amarillo claro sobre las fibras. El cambio de $\mathrm{pH}$ produjo tonalidad beige, café y amarillo claro, amarillo y verde, amarillo mostaza, naranja claro, café rojizo y verde claro con Chlorophyllum molybdites, Earliella scabrosa, Phellinus robustus y Ganoderma aff. lucidum registrados por primera vez como tintóreos. Los hongos son una buena alternativa para la obtención de tintes naturales.

Palabras clave: hongos tintóreos, fibras vegetales, fibras animales, colorantes naturales

\section{ABSTRACT}

Background: Natural dyes have been used since pre-Hispanic times. However, there is little literature on the extraction of fungal dyes to dye organic fibers.

Objective: To evaluate the dyeing potential of five wild fungi on fibers of plant and animal origin.

Methods: The fungi were fragmented, hydrated $24 \mathrm{~h}$, and boiled for $45 \mathrm{~min}$ to obtain the dyes. Linen, henequen, scourer, hemp, and wool fibers were used to evaluate the effectiveness of the dye. The fibers were washed with neutral soap, mordanting, and boiled for $1 \mathrm{~h}$ in the dye. In a second experiment, the $\mathrm{pH}$ of the dyes was modified with vinegar, sodium carbonate and ammonia to test new colors on the fibers.

Results and conclusions: The dyes showed dark reddish brown to light yellow brown hues on the fibers. The pH change produced beige, brown and light yellow, yellow and green, mustard yellow, light orange, reddish brown and light green hue with Chlorophyllum molybdites, Earliella scabrosa, Phellinus robustus and Ganoderma aff. lucidum recorded for the first time as dyes. Mushrooms are a good alternative for obtaining natural dyes.

Keywords: dyeing fungi, vegetable fibers, animal fibers, natural colorants

\section{ARTICLE HISTORY}

Received: 03/June/2021. Accepted: 08/September/2021

Published on line: 08/September/2021

\section{CORRESPONDING AUTHOR}

W Silvia Cappello, cappellogs@hotmail.com

ORCID: 0000-0003-1354-6304 


\section{INTRODUCCIÓN}

Los hongos son un componente vital en el funcionamiento de los ecosistemas debido a que contribuyen de manera significativa en el desarrollo de las poblaciones vegetales y animales (Villarruel-Ordaz et al. 2015). Desde la antigüedad, estos organismos fueron útiles para el ser humano, siendo recolectados para la alimentación, la supervivencia y la economía (Boa 2005). En la actualidad los hongos forman parte de diversas actividades biotecnológicas, entre las que se encuentra la obtención de tintes naturales para la tinción de fibras textiles de origen vegetal y animal, la cual se ha venido trabajando sobre todo a nivel artesanal (Acosta-Urdapilleta et al. 2012; Portillo et al. 2019).

El estudio de los hongos tintóreos está representado por dos líneas de investigación. La primera, abarca la composición química de los pigmentos hallados en algunas especies de Basidiomycetes, donde dichas sustancias naturales son las encargadas de dar color a los carpóforos (Vigueras y Portillo 2016; Portillo et al. 2019), incluyendo a diversas especies de macromicetos de zonas templadas (Zhou y Liu 2010; Velišek y Cejpek 2011). La segunda línea de investigación comprende la extracción de los colorantes naturales que se encuentran en los hongos. Diversas especies fúngicas en sus diferentes grupos son una inmensa fuente de colorantes, tal como lo describen Vigueras y Portillo (2016) quienes indicaron que la intensidad del teñido puede variar dependiendo de la especie fúngica, además mencionan algunas especies de interés con su respectiva coloración, entre ellos Hypomyces lactifluorum que tiñe de color amarillo, naranja y marrón rojizo, Polyporus schweinitzii que tiñe de amarillo oro, marrón oscuro y verde, Amanita caesarea que tiñe colores muy tenues como amarillo paja y gris, entre otros.

A nivel nacional el estudio de los hongos tintóreos es escaso, se han evaluado con frecuencia en especies de poliporales (Cedano et al. 2001; Cedano y Villaseñor 2006). Vigueras y Portillo (2013) extrajeron el pigmento quinónico a través de la técnica Sohxlet, de Ganoderma sessile, con el cual tiñeron un detergente, sugiriendo la posibilidad de utilizar estos colorantes en diversas áreas de la industria, como la tinción de productos de limpieza.

El uso de los hongos como tintes orgánicos es reciente, a escala mundial se han reportado cerca de 126 especies con potencial tintóreo, utilizados en la tinción de fibras. Lo anterior muestra que aún existe una cantidad ingente de taxones por valorar su contenido y potencial como colorante orgánico en los diversos campos de la industria (Cedano y Villaseñor 2006).

En la actualidad existe la necesidad de emplear tintes naturales para la coloración de textiles, debido a que estos colorantes son renovables y biodegradables, y tienen una mayor compatibilidad con el medio ambiente en comparación con los tintes sintéticos (Anchana 2014). Además, los tintes naturales tienen demanda en la coloración de alimentos, cosméticos y medicamentos, con la finalidad de sustituir sustancias químicas dañinas empleadas en la elaboración de dichos productos (Velmurugan et al. 2010).

Los tintes naturales provenientes de productos maderables y no maderables datan desde la antigüedad por su uso tradicional, sin embargo, el progreso y las técnicas de industrialización han ocasionado el reemplazo de dichos tintes por aquellos catalogados como "sintéticos" (Carmona 2013; Portillo et al. 2019). Estos tintes artificiales están compuestos de sustancias químicas con repercusiones negativas para el medio natural y daños a la salud humana (Anchana 2014; Velmurugan et al. 2010). Entre las consecuencias está la contaminación del agua y del ambiente (Vigueras y Portillo 2016), así como enfermedades cancerígenas, lesiones, desequilibrio en el sistema renal y enfermedades respiratorias (Rojas et al. 2011).

Lo anterior ha generado el regreso en el uso de los tintes naturales a nivel industrial (farmacéutica, alimenticia, cosmética, entre otras) debido a que no representan una amenaza para el medio ambiente (SEMARNAT 2013), un ejemplo claro es la utilización del pigmento rojo-púrpura producido por Gibberella zeae (Fusarium graminearum) para el control de diversas bacterias de interés clínico (Villanueva-Arce et al. 2013).

Otro caso es el hongo Monascus sp., el cual produce pigmentos amarillos (monascina y ankaflavina), naranjas (monascorubrín y rubropuctatín) y rojos (monascorubramina y rubropuctamina) usados para colorear arroz, vino, queso, pescado y carnes (Reyes-González y Franco-Correa 2006). Los tintes naturales no son nocivos para el ser humano, y poseen propiedades como estabilidad a la luz y a tratamientos térmicos, y son de fácil manejo, por lo tanto tienen cualidades atractivas para el sector textil y artesanal (Vigueras y Portillo 2016). 
El conocimiento de los hongos tintóreos en México no se ha documentado a gran escala, sin embargo, representa una alternativa de aprovechamiento sustentable, por tal razón es importante estudiar el potencial tintóreo de los macromicetos, y abundar en estudios que permitan optimizar el proceso de extracción de tintes y teñido de fibras. Por lo tanto, el objetivo de esta investigación fue evaluar el potencial tintóreo de cinco especies de hongos macroscópicos nativos de Tabasco, sobre fibras de origen vegetal y animal.

\section{MATERIALES Y MÉTODOS}

El material de estudio pertenece a la Colección de Hongos del Herbario de la División Académica de Ciencias Biológicas - Universidad Juárez Autónoma de Tabasco (UJAT). Las especies utilizadas fueron Chlorophyllum molybdites Massee (Cappello-García 3050), Earliella scabrosa Gilb. \& Ryvarden (Cappello-García 49), Ganoderma aff. lucidum P. Karst. (Marín 1195), Phellinus robustus Bourdot \& Galzin (Espinoza-lbarra 1) y Pycnoporus sanguineus Murrill (Díaz-Contreras 173). Las fibras empleadas fueron seleccionadas de acuerdo a la disponibilidad del recurso en la región, empleando henequén (Agave fourcroydes Lem), cáñamo (Cannabis sativa L.), lino (Linum usitatissimum L.) estropajo (Luffa cylindrica L.) y lana (Ovis orientalis L.) delgada y gruesa. En el caso de los mordientes, para las fibras vegetales se utilizó alumbre (sulfato alumínico potásico KAl $\left.\left(\mathrm{SO}_{4}\right) 2 \cdot 12 \mathrm{H}_{2} \mathrm{O}\right)$, carbonato de sodio $\left(\mathrm{Na}_{2} \mathrm{CO}_{3}\right)$ y semilla de aguacate (Persea americana P. Mill.) como tanino. Para las fibras animales se utilizó crémor tártaro $\left(\mathrm{KC}_{4} \mathrm{H}_{5} \mathrm{O}_{6}\right)$ y alumbre.

\section{Preparación de las fibras y premordentado}

La preparación de las fibras se realizó bajo la metodología de Rice (1990), con algunas modificaciones de Vigueras y Portillo (2016). Por cada fibra se emplearon siete piezas de nueve centímetros de largo. Éstas se agruparon en forma de madejas, se lavaron con jabón neutro y se enjuagaron a fin de retirar la grasa y otros compuestos que pudieron hallarse sobre la superficie de las fibras, luego se secaron y se pesaron. Tres baños de mordiente fueron aplicados para el premordentado de las fibras vegetales, en las que se empleó dos litros de agua por cada mordiente. El alumbre, carbonato de sodio y tanino, fueron usados con proporciones de 20 $\%, 5 \%$ y $25 \%$ respectivamente. Dichas proporciones se calcularon con base al peso seco de la fibra vegetal (84.65 g).

Para realizar el premordentado de la fibra se empleó un recipiente de peltre. Para el primer baño se disolvió el carbonato de sodio con el alumbre en dos litros de agua, luego con ayuda de una parrilla eléctrica se calentó a una temperatura promedio de $30{ }^{\circ} \mathrm{C}$ donde se introdujeron las fibras húmedas manteniéndolas en ebullición durante una hora. Después la mezcla se dejó enfriar y al día siguiente se extrajeron las fibras, al final del proceso se lavaron con jabón neutro, se enjuagaron y se dejaron secar. El segundo baño fue similar al primero pero se empleó el tanino como mordiente. El tercer baño se realizó igual que el primero pero adicionando $5 \%$ más de alumbre.

El premordentado de la fibra animal fue similar al de las fibras vegetales, se calculó con base al peso seco de la fibra $(28.80 \mathrm{~g})$ con proporciones de $2.77 \%$ de crémor tártaro y $20 \%$ de alumbre. La cantidad de crémor tártaro fue tomado del documento "Tinturas forestales y su uso en el teñido de fibras naturales" (SEMARNAT 2013). Ambos ingredientes se disolvieron en dos litros de agua y junto con las fibras se mantuvieron en ebullición durante una hora. Las fibras se dejaron enfriar hasta el día siguiente y posterior fueron lavadas con jabón neutro.

\section{Extracción del tinte y teñido de las fibras}

La preparación de los tintes se realizó de la siguiente manera. Por cada especie fúngica empleada se pesaron $60 \mathrm{~g}$ de basidioma seco, y luego se realizaron fragmentos de $2 \mathrm{~cm}$, los cuales se hidrataron durante $24 \mathrm{~h}$ en un recipiente con $2 \mathrm{~L}$ de agua. Transcurrido el tiempo la mezcla se calentó sobre una parrilla eléctrica dejándola hervir durante 45 min, luego se dejó enfriar y al día siguiente la mezcla de colorante se filtró con un colador. Las fibras vegetales y animales tratadas con anterioridad, se introdujeron por separado en el tinte obtenido por cada especie fúngica. El tinte junto con las fibras se calentaron a punto de ebullición durante 1 $\mathrm{h}$ en un recipiente de peltre, $\mathrm{y}$ al día siguiente las fibras fueron retiradas y secadas a temperatura ambiente.

\section{Evaluación del potencial tintóreo}

Para describir la gama de colores que cada tinte pudiera proporcionar a las fibras se realizó una segunda fase experimental, la cual consistió en modificar el pH de los tintes de cada hongo con tres sustancias dife- 
rentes, vinagre, solución de carbonato de sodio (al 90 \%) y amoniaco $\left(\mathrm{NH}_{3}\right)$ en dos proporciones. Este último se empleó como control de acuerdo a Rice (1990). Esta fase experimental sólo se le aplicó a la fibra de lana debido a que en la etapa previa presentó mayor fijación del tinte. Para cada sustancia empleada se trabajó de manera similar. La prueba consistió en colocar $85 \mathrm{~mL}$ de tinte en un recipiente de vidrio, agregarle $5 \mathrm{~mL}$ de amoniaco, registrar el cambio de color del tinte por el $\mathrm{pH}$, e introducir en la mezcla tres madejas pequeñas de fibra. Para el teñido de las fibras la mezcla se mantuvo 45 min a baño María. El proceso fue repetido añadiendo otros $5 \mathrm{~mL}$ de amoniaco.

\section{Pruebas de resistencia de lavado}

Cada fibra vegetal y animal teñida fue lavada con agua potable y detergente comercial para conocer la resis- tencia del color obtenido. Los colores derivados de los procesos antes descritos (tanto de tintes como de fibras) se identificaron con la Guía de Colores de Küppers (1996). Es importante recomendar que sólo se usen ollas de peltre bien conservadas, ya que no interfieren en el color del tinte como lo serian las de aluminio y cobre, asimismo se recomienda palas de madera para mover las fibras.

\section{RESULTADOS Y DISCUSIÓN}

Por cada hongo utilizado se obtuvieron alrededor de $1.5 \mathrm{~L}$ de tinte, en especial tonalidades de color café con ligeras variaciones (Tabla 1).

De las especies utilizadas $P$. sanguineus se ha reportado como tintórea en estudios previos (Acosta-Urdapilleta et al. 2010; Cruz et al. 2015), y la coloración obtenida

Tabla 1. Tonalidad de los tintes extraídos por cada especie

\begin{tabular}{|c|c|c|}
\hline Especie & Tono del tinte & Tinte \\
\hline Chlorophyllum molybdites & Café amarillento (N50, A90, M50) & \\
\hline Earliella scabrosa & Café rojizo (N40, A90, M60) & \\
\hline Ganoderma aff. lucidum & Café anaranjado (N50, A99, M50) & \\
\hline Phellinus robustus & Café claro (N60, A90, M50) & \\
\hline Pycnoporus sanguineus & Café oscuro (N40, A99, M70) & \\
\hline
\end{tabular}


corresponde a la registrada en este trabajo. Algunas especies de Ganoderma y Phellinus figuran dentro de dichos reportes con tonalidades de color café, similares a este reporte (Cedano y Villaseñor 2006). La capacidad tintórea de $C$. molybdites y $E$. scabrosa no se sustenta en estudios previos, ya que estos hongos son de zonas tropicales y la mayoría de los trabajos en la extracción de tintes se ha realizado en zonas templadas (Cedano y Villaseñor 2006; Zhou y Liu 2010; Velišek y Cejpek 2011; Franco-Maass et al. 2019). Ambas especies produjeron tonalidades de color café amarillento y café rojizo, ésta última muy similar a los tonos registrados en las especies del género Phellinus (Cedano y Villaseñor 2006).

\section{Tonalidades en las fibras animal y vegetal}

Las coloraciones obtenidas fueron más claras en las fibras vegetales, a diferencia de la fibra animal que presentó tonalidades con mayor intensidad (Tabla 2). Cabe señalar que el color natural de las fibras vegetales utilizadas en el proceso de teñido fue de blanco en

Tabla 2. Tonalidades obtenidas en las fibras de origen vegetal y animal con diferentes especies de hongos

\begin{tabular}{|c|c|c|c|c|c|c|}
\hline Especie & Cáñamo & Estropajo & Henequén & Lino & Lana gruesa & Lana delgada \\
\hline $\begin{array}{l}\text { Chlorophyllum } \\
\text { molybdites }\end{array}$ & $\begin{array}{c}\text { Café claro } \\
\text { (N30A70M50) }\end{array}$ & $\begin{array}{l}\text { Beige obscuro } \\
\text { (N30A60M30) }\end{array}$ & $\begin{array}{l}\text { Amarillo claro } \\
\text { (N30A70M30) }\end{array}$ & $\begin{array}{c}\text { Beige claro } \\
\text { (N30A40M20) }\end{array}$ & $\begin{array}{c}\text { Beige } \\
\text { (N30A50M20) }\end{array}$ & $\begin{array}{c}\text { Café } \\
\text { (N30A70M30) }\end{array}$ \\
\hline Earliella scabrosa & $\begin{array}{c}\text { Café } \\
\text { (N30A99M50) }\end{array}$ & $\begin{array}{c}\text { Café claro } \\
\text { (N30A70M30) }\end{array}$ & $\begin{array}{c}\text { Café claro } \\
\text { (N30A90M40) }\end{array}$ & $\begin{array}{c}\text { Beige claro } \\
\text { (N30A60M30) }\end{array}$ & $\begin{array}{c}\text { Café claro } \\
\text { (N40A70M50) }\end{array}$ & $\begin{array}{c}\text { Café } \\
\text { (N40A99M50) }\end{array}$ \\
\hline Ganoderma lucidum & $\begin{array}{c}\text { Café claro } \\
\text { (N20A80M50) }\end{array}$ & $\begin{array}{c}\text { Beige (N20A30 } \\
\text { M20) }\end{array}$ & $\begin{array}{l}\text { Amarillo claro } \\
\text { (N20A60M30) }\end{array}$ & $\begin{array}{c}\text { Beige claro } \\
\text { (N20A50M30) }\end{array}$ & $\begin{array}{l}\text { Mostaza claro } \\
\text { (N20A99M30) }\end{array}$ & $\begin{array}{l}\text { Mostaza claro } \\
\text { (N20A99M30) }\end{array}$ \\
\hline Phellinus robustus & $\begin{array}{l}\text { Café obscuro } \\
\text { (N10A90M50) }\end{array}$ & $\begin{array}{c}\text { Beige } \\
\text { (N10A50M40) }\end{array}$ & $\begin{array}{l}\text { Mostaza claro } \\
\text { (A40M30C10) }\end{array}$ & $\begin{array}{l}\text { Amarillo claro } \\
\text { (N10A40M20) }\end{array}$ & $\begin{array}{c}\text { Mostaza } \\
\text { (N40A99M50) }\end{array}$ & $\begin{array}{c}\text { Mostaza } \\
\text { (N40A99M50) }\end{array}$ \\
\hline $\begin{array}{l}\text { Pycnoporus } \\
\text { sanguineus }\end{array}$ & $\begin{array}{c}\text { Café rojizo } \\
\text { (N40A99M60) }\end{array}$ & $\begin{array}{c}\text { Marrón } \\
\text { (N20A60M40) }\end{array}$ & $\begin{array}{l}\text { Marrón claro } \\
\text { (N20A90M50) }\end{array}$ & $\begin{array}{l}\text { Marrón claro } \\
\text { (N20A70M40) }\end{array}$ & $\begin{array}{c}\text { Café rojizo } \\
\text { (A60M70C60) }\end{array}$ & $\begin{array}{c}\text { Café rojizo } \\
(\mathrm{A} 60 \mathrm{M} 70 \mathrm{C} 70)\end{array}$ \\
\hline
\end{tabular}


el lino a tonos beige en el estropajo y henequén, así como café en el cáñamo (Küppers 1996).

Respecto a la fibra animal la coloración inicial de la lana fue blanca, en este sentido ofreció mayor ventaja de fijación a los tintes fúngicos (Tabla 2) con tonalidades más intensas. La coloración inicial de la lana le ha permitido ser una de las fibras con mayor utilidad en su teñido sobre todo a base de plantas (frutos, raíces, hojas, corteza, flores, etc.) y algunos insectos (Franco-Maass et al. 2019). En México es utilizada para artesanías textiles tales como la elaboración de diferentes prendas de vestir. Posee características que la convierten en una materia prima inigualable al obtener productos de alta calidad, entre las características de la fibra está la conducción de calor, aislación térmica, manchado, limpieza, elongación, resistencia al calor y a la inflamabilidad. La lana tiene propiedades higroscópicas, cualidad que le permite absorber hasta el 50 $\%$ de su peso en agua, y dicha cualidad le facilita un mayor teñido (Elvira 2009).

Tabla 3. Potencial tintóreo de los hongos con base en el cambio de pH

\begin{tabular}{|c|c|c|c|c|c|c|c|}
\hline \multirow[b]{2}{*}{ Especie } & \multirow[b]{2}{*}{$\begin{array}{c}\mathrm{pH} \\
\text { inicial }\end{array}$} & \multicolumn{2}{|c|}{ Bicarbonato } & \multicolumn{2}{|c|}{ Vinagre blanco } & \multicolumn{2}{|c|}{ Amoniaco } \\
\hline & & C1 & $\mathrm{C} 2$ & $\mathrm{C} 1$ & $\mathrm{C} 2$ & C1 & C2 \\
\hline $\begin{array}{l}\text { Chlorophyllum } \\
\text { molybdites }\end{array}$ & 5.2 & $\begin{array}{c}8.9 \\
\text { Beige amarillento } \\
\text { (N20A50M20) }\end{array}$ & $\begin{array}{c}10.3 \\
\text { Amarillo naranja } \\
\text { (N20A80M30) }\end{array}$ & $\begin{array}{c}4.2 \\
\text { Café claro } \\
\text { (N30A99M40) }\end{array}$ & $\begin{array}{c}4.0 \\
\text { Amarillo verdoso } \\
\text { (N30A70M20) }\end{array}$ & $\begin{array}{c}10.3 \\
\text { Beige verdoso ce- } \\
\text { nizo (N40A60M30) }\end{array}$ & $\begin{array}{c}10.1 \\
\text { Verde amarillento } \\
\text { (N30A90M30) }\end{array}$ \\
\hline $\begin{array}{l}\text { Earliella sca- } \\
\quad \text { brosa }\end{array}$ & 5.5 & $\begin{array}{c}10.5 \\
\text { Café verdoso } \\
\text { (N40A90M50) }\end{array}$ & $\begin{array}{c}9.7 \\
\text { Amarillo marrón } \\
\text { (N50A99M50) }\end{array}$ & $\begin{array}{c}4.0 \\
\text { Café claro verdoso } \\
\text { (N60A80M40) }\end{array}$ & $\begin{array}{c}6.8 \\
\text { Café claro } \\
\text { (N50A70M30) }\end{array}$ & $\begin{array}{c}10.7 \\
\text { Verde olivo cenizo } \\
\text { (N60A50M20) }\end{array}$ & $\begin{array}{c}10.7 \\
\text { Verde grisáceo } \\
\text { (N50A50M20) }\end{array}$ \\
\hline $\begin{array}{l}\text { Ganoderma } \\
\text { lucidum }\end{array}$ & 5.5 & $\begin{array}{c}10.2 \\
\text { Beige verdoso cla- } \\
\text { ro (N40A50M20) }\end{array}$ & $\begin{array}{c}10.3 \\
\text { Naranja opaco } \\
\text { (N30A99M40) }\end{array}$ & $\begin{array}{c}3.8 \\
\text { Marrón claro } \\
\text { (N40A99M50) }\end{array}$ & $\begin{array}{c}3.5 \\
\text { Amarillo oscuro } \\
\text { (N40A99M40) }\end{array}$ & $\begin{array}{c}10.7 \\
\text { Beige amarillento } \\
\text { (N40A40M20) }\end{array}$ & $\begin{array}{c}10.3 \\
\text { Beige oscuro } \\
\text { (N40A50M10) }\end{array}$ \\
\hline $\begin{array}{l}\text { Phellinus ro- } \\
\text { bustus }\end{array}$ & 5.8 & $\begin{array}{c}10.7 \\
\text { Amarillo pálido } \\
\text { (N10A40M20) }\end{array}$ & $\begin{array}{c}10.3 \\
\text { Café claro verdoso } \\
\text { (N40A70M30) }\end{array}$ & $\begin{array}{c}3.7 \\
\text { Café claro } \\
\text { (N40A99M50) }\end{array}$ & $\begin{array}{c}3.3 \\
\text { Café chocolate cla- } \\
\text { ro (N40A80M40) }\end{array}$ & $\begin{array}{c}11.2 \\
\text { Café verdoso claro } \\
\text { (N40A60M30) }\end{array}$ & $\begin{array}{c}10.6 \\
\text { Amarillo } \\
\text { mostaza claro }\end{array}$ \\
\hline $\begin{array}{l}\text { Pycnoporus } \\
\text { sanguineus }\end{array}$ & 4.7 & $\begin{array}{c}10.2 \\
\text { Café claro } \\
\text { (N60A90M40) }\end{array}$ & $\begin{array}{c}10.2 \\
\text { Verde amarillento } \\
\text { (N50A80M30) }\end{array}$ & $\begin{array}{c}3.5 \\
\text { Café rojizo } \\
\text { (N50A99M50) }\end{array}$ & $\begin{array}{c}3.6 \\
\text { Café amarillento } \\
\text { (N40A80M40) }\end{array}$ & $\begin{array}{c}10.6 \\
\text { Verde amarillento } \\
\text { (N60A90M10) }\end{array}$ & $\begin{array}{c}10.6 \\
\text { Café grisáceo } \\
\text { (N70A60M20) }\end{array}$ \\
\hline
\end{tabular}


Respecto a los mordientes usados en la preparación de las fibras se emplearon aquellos que por lo regular son utilizados en los procesos de tinturas por hongos en fibras tanto animales como vegetales (Vigueras y Portillo 2016), se eligieron aquellos disponibles en la región y de fácil acceso, ya que algunos pueden encontrarse en los mercados locales. Los mordientes están asociados a propiedades benéficas para el humano, en el caso del alumbre se recomienda como antitranspiran- te, antimicótico y antibacteriano sin repercusiones para la piel; la semilla de aguacate ha sido señalada con propiedades medicinales (Méndez y Alva 2018).

\section{Evaluación del potencial tintóreo}

El pH inicial de los tintes obtenidos a partir de las especies fúngicas registró un valor de 4.7 a 5.5 con una tendencia ácida. Estos valores en presencia del vinagre variaron de 3.3. a 6.8 manteniendo la tendencia ácida;

Tabla 4. Apariencia de las fibras teñidas después del lavado con detergente comercial

\begin{tabular}{|c|c|c|c|c|c|c|}
\hline Especie & Cáñamo & Estropajo & Henequén & Lino & Lana gruesa & Lana delgada \\
\hline $\begin{array}{l}\text { Chlorophyllum } \\
\text { molybdites }\end{array}$ & $\begin{array}{c}\text { Café claro } \\
\text { (N40A90M50) }\end{array}$ & Beige (N30A40M20) & $\begin{array}{l}\text { Verde olivo opaco } \\
\text { (N30A60M20) }\end{array}$ & $\begin{array}{l}\text { Beige amarillento } \\
\text { (N20A20M10) }\end{array}$ & $\begin{array}{l}\text { Amarillo mostaza pá- } \\
\text { lido (N20A50M20) }\end{array}$ & $\begin{array}{l}\text { De beige a rosa pá- } \\
\text { lido (N20A40M20) }\end{array}$ \\
\hline Earliella scabrosa & $\begin{array}{l}\text { Café verdoso } \\
\text { (N40A99M30) }\end{array}$ & $\begin{array}{l}\text { Café verdoso claro } \\
\text { (N40A60M30) }\end{array}$ & $\begin{array}{c}\text { Café claro } \\
\text { (N40A60M30) }\end{array}$ & $\begin{array}{l}\text { Amarillo claro } \\
\text { (N10A20M10) }\end{array}$ & $\begin{array}{l}\text { Café opaco } \\
\text { (N50A99M40) }\end{array}$ & $\begin{array}{l}\text { Café verdoso } \\
\text { (N50A90M40) }\end{array}$ \\
\hline $\begin{array}{l}\text { Ganoderma } \\
\text { lucidum }\end{array}$ & $\begin{array}{c}\text { Café claro } \\
\text { (N40A80M40) }\end{array}$ & $\begin{array}{l}\text { Amarillo pálido } \\
\text { (N10A10M10) }\end{array}$ & $\begin{array}{l}\text { Amarillo opaco } \\
\text { (A50M30C30) }\end{array}$ & $\begin{array}{c}\text { De amarillo claro a } \\
\text { beige (N10A20M10) }\end{array}$ & $\begin{array}{l}\text { Café verdoso claro } \\
\text { (N40A90M50) }\end{array}$ & $\begin{array}{l}\text { Amarillo mostaza } \\
\text { (N40A99M40) }\end{array}$ \\
\hline Phellinus robustus & $\begin{array}{c}\text { Café claro } \\
\text { (N30A99M40) }\end{array}$ & $\begin{array}{l}\text { Amarillo cremita } \\
\text { (N10A30M20) }\end{array}$ & $\begin{array}{l}\text { Beige amarillento } \\
\text { (N10A30M20) }\end{array}$ & $\begin{array}{l}\text { Amarillo pálido } \\
\text { (N10A20M10) }\end{array}$ & $\begin{array}{l}\text { Amarillo mostaza } \\
\text { (N50A99M30) }\end{array}$ & $\begin{array}{c}\text { Amarillo mostaza } \\
\text { (N50A99M40) }\end{array}$ \\
\hline $\begin{array}{l}\text { Pycnoporus } \\
\text { sanguineus }\end{array}$ & $\begin{array}{l}\text { Café chocolate } \\
\text { (N60A80M50) }\end{array}$ & $\begin{array}{l}\text { Verde olivo oscuro } \\
\text { (N30A90M30) }\end{array}$ & $\begin{array}{c}\text { Café verdoso claro } \\
\text { (N50A99M40) }\end{array}$ & $\begin{array}{l}\text { Café con leche } \\
\text { (N40A60M30) }\end{array}$ & $\begin{array}{l}\text { Café oscuro } \\
\text { (N60A80M50) }\end{array}$ & $\begin{array}{l}\text { Café oscuro } \\
\text { (N60A99M50) }\end{array}$ \\
\hline
\end{tabular}


el pH en presencia de bicarbonato y amoniaco registró valores de 8.9 a 10.7 y 10.1 a 11.2 respectivamente, en este caso la tendencia fue alcalina.

En trabajos previos el amoniaco tiene la capacidad de modificar el $\mathrm{pH}$ de los tintes de manera significativa, logrando obtener una amplia gama de coloraciones o tonalidades (Rice 1990). Sin embargo, en este trabajo el amoniaco se empleó como testigo debido a los efectos nocivos (corrosiva e irritante) que puede provocar durante la manipulación. En ese sentido el bicarbonato de sodio puede constituir su remplazo, logrando valores de $\mathrm{pH}$ similares. En lo que respecta al vinagre no se reportan daños nocivos durante su manipulación para el empleo del teñido de fibras.

Cabe resaltar que el cambio de $\mathrm{pH}$ en los tintes constituye una técnica importante para explorar el potencial tintóreo en los hongos debido a los cambios en las tonalidades según las especies utilizadas, esta práctica se realizó en los primeros trabajos de Rice (1990) sin embargo, en estudios recientes no se ha reportado. En este estudio para la fibra de lana se obtuvo en $C$. molybdites tonos beige a amarillo claro. E. scabrosa presentó coloraciones de café claro a tonos amarillos y verdes. Ganoderma aff. lucidum mostró tonos de amarillo mostaza claro a naranja claro, PheIlinus robustus tonos de amarillo mostaza a amarillo, y Pycnoporus sanguineus de café claro a café rojizo y verde claro (Tabla 3).

La resistencia del color en las fibras vegetales y animales después de su teñido es alta pese a su lavado con detergentes comerciales, éstas tienden a mantener su coloración con ligeras disminuciones en su apariencia (Tabla 4). Este último punto es importante para discernir en el uso de fibras afines al teñido de los hongos ante una alternativa comercial.

\section{CONCLUSIONES}

El presente trabajo, es el primer registro en el uso potencial de hongos tropicales para la extracción de tintes naturales, en este sentido las especies trabajadas resultaron viables para la tinción de fibras tanto animales como vegetales. Por lo general las tonalidades de los tintes de los hongos tropicales son de amarillo, café y beige. Las fibras tanto animales como vegetales mantienen el color aún después de varios procesos de lavado con detergentes comerciales. El potencial de los hongos tintóreos sobre las fibras naturales en Méxi- co, es importante para la industria y el sector artesanal, que ha sido poco aprovechado.

\section{AGRADECIMIENTOS}

Los autores agradecen a la División Académica de Ciencias Biológicas de la Universidad Juárez Autónoma de Tabasco por el espacio brindado para la realización del trabajo.

\section{LITERATURA CITADA}

Acosta-Urdapilleta L, Alonso-Paz GA, Rodríguez A, Adame M, Salgado D, Montiel-Peña M, Medrano-Vega F, Villegas-Villarreal EC. 2010. Pycnoporus sanguineus, un hongo con potencial biotecnológico In: Martínez-Carrera D, Cuvetto N, Sobal M, Morales P, Mora VM (eds.) Hacia un desarrollo sustentable de producción-consumo de los hongos comestibles y medicinales en Latinoamérica: avances y perspectivas en el siglo XXI. Red Latinoamericana de Hongos Comestibles y Medicinales. COLPOS-UNS-CONACYT-AMC-UAEMUPAEP-IMINAP. 531-562.

Acosta-Urdapilleta L, Medrano VFA, Villegas VEC. 2012. Pigmentos antimicrobianos de Pycnoporus sanguineus. In: Sánchez-Vásquez JE, Mata G (eds.) Hongos comestibles y medicinales en Iberoamérica. Investigación y desarrollo en un entorno multicultural. ECOSUR-INECOL, Tapachula. 339-347.

Anchana DA. 2014. Extraction of natural dyes from fungus - an alternate for textile dyeing. Journal of Natural Sciences Research $4,1-6$.

Boa E. 2005. Los hongos silvestres comestibles, perspectiva global de su uso e importancia para la población, productos forestales no madereros. Organización de las Naciones Unidas para la Agricultura y la Alimentación, Roma.

Carmona I. 2013. De colorantes sintéticos a naturales en la industria alimentaria. Odepa Santiago de Chile 5: 1-6.

Cedano M, Villaseñor IL, Guzmán DL. 2001. Some Aphyllophorales tested for organic dyes. Mycologist 15, 81-85. https://doi. org/10.1016/S0269-915X(01)80089-6

Cedano M, Villaseñor IL. 2006. Colorantes orgánicos de hongos y líquenes. Revista Scientia-CUCBA 8, 141-161.

Cruz MR, Piña-Guzmán AB, Yáñez-Fernández J, Valencia-Del Toro G, Bautista-Baños S, Villanueva AR. 2015. Producción de pigmentos de Pycnoporus sanguineus en medio de cultivo sólido. Agrociencia 49, 347-359.

Elvira MG. 2009. De que está hecha la lana y principales características textiles. Sitio Argentino de Producción Animal. Carpeta Técnica, Ganadería Nº33, EEA INTA, Esquel.

Franco-Maass S, Burrola-Aguilar C, Arana-Gabriel Y, Arredondo-Ayala G, Cruz-Balderas Y. 2019. Conocimiento local y uso potencial tintóreo de hongos comestibles en el poniente del Estado de México. Agroproductividad 12, 37-43. https://doi.org/10.32854/ agrop.v0i0.1394

Küppers H. 1996. Atlas de los colores. Editorial Blume, Barcelona.

Méndez GBY, Alva MPN. 2018. Efecto del extracto hexanoico de la semilla del aguacate criollo mexicano sobre Trichomonas vaginalis. Jovenes en la Ciencia 4, 698-702.

Portillo L, Julián A, Santana M. 2019. Fibras naturales y pigmentos: teñido, tejido y diseño. Centro Universitario de Ciencias Biológi- 
cas y Agropecuarias, Centro Universitario de Arte, Arquitectura y Diseño, Universidad de Guadalajara, México. Edición Especial del Boletín Nakari 30, Núm. 3.

Reyes-González G, Franco-Correa M. 2006. Producción biotecnológica de sabores, pigmentos y aromas a partir de hongos miceliales y levaduras. Universitas Scientiarum 11, 23-30.

Rice MC. 1990. Mushrooms for color. In Dyes from Nature. Plants \& Gardens, Brooklyn Botanic Garden Record 46, 42-45.

Rojas LO, Mavila HD, Rojas PN. 2011. Insumos ecológicos en la serigrafía textil: caso peruano. Industrial Data 14, 34-41.

SEMARNAT, 2013. Tinturas forestales y su uso en el teñido de fibras naturales. https://www.google.com.mx/search?q=Tinturas+forestales+y+su+uso+en+el+te\%C3\%B1ido+de+fibras+naturales+(SEMARNAT+WWW. CONAFOR.GOB. $M X) \& o q=$ Tinturas + forestales $+y+$ su + uso $+e n+e l+$ te $\% C 3 \%-$ $\mathrm{B} 1 \mathrm{ido}+\mathrm{de}+$ fibras + naturales+(SEMARNAT+WWW.CONAFOR.GOB.MX)\&aqs=chrome..69i57.633j0j4\&sourceid=chrome\&ie=UTF-8 (12 de junio del 2015).

Velišek J, Cejpek K. 2011. Pigments of higher fungi - a review. Czech Journal of Food Sciences 29, 87-102. https://doi.org/10.17221/524/2010-CJFS

Velmurugan P, Kamala-Kannan S, Balachandar V, Lakshmanaperumalsamy $P$, Chae J, Oh B. 2010. Natural pigment extraction from five filamentous fungi for industrial applications and dyeing of leather. Carbohydrate Polymers 79, 262-268. https://doi.org/10.1016/j.carbpol.2009.07.058

Vigueras AL, Portillo L. 2013. Teñido de fibras con grana cochinilla y otros colorantes naturales. Cactusnet Newsletter 13, 165-175.

Vigueras AL, Portillo L. 2016. Teñido de fibras naturales con grana cochinilla del nopal y otros pigmentos. In: Vigueras AL, Portillo L. (eds.) Conocimiento y aprovechamiento de cactáceas y otras plantas suculentas. Grana cochinilla del nopal y otros pigmentos en el teñido de fibras naturales. Universidad de Guadalajara, Guadalajara. 9-35.

Villanueva-Arce R, Aguilar-Pompa CA, Gómez y Gómez YM, Valencia-Del Toro G, Piña-Guzmán AB, Bautista-Baños S. 2013. Control de bacterias patógenas y hongos de postcosecha con extractos del pigmento de Gibberella zeae (Fusarium graminearum). Agrociencia 47: 691-705.

Villarruel-Ordaz JL, Canseco ZE, Cifuentes J. 2015. Diversidad fúngica en el municipio de San Gabriel Mixtepec, región Costa de Oaxaca, México. Revista Mexicana de Micología 41, 55-63.

Zhou Z, Liu J. 2010. Pigments of fungi (macromycetes). Natural Product Reports. Journal of the Royal Society of Chemistry 11, 15131724. https://doi.org/10.1039/c004593d 